\title{
GPPS-BJ-2019-0059
}

\section{Laminar flame speeds of methane/air mixture at engine-relevant conditions: performance of different mechanisms}

\author{
Yiqing Wang \\ Peking University \\ wangyiqing@pku.edu.cn \\ Beijing, China
}

\author{
Jingyi Su \\ Peking University \\ 1801111628@pku.edu.cn \\ Beijing, China
}

\author{
Zheng Chen \\ Peking University \\ cz@pku.edu.cn \\ Beijing, China
}

\begin{abstract}
Laminar flame speed (LFS) is used as an important input in certain turbulent premixed combustion modelling for spark ignition engines. At engine-relevant temperatures and pressures, the LFS is difficult to be measured and it is usually obtained from calculations using detailed or reduced chemical mechanisms. However, at very high temperature in the range of $700 \sim 1000 \mathrm{~K}$, it is difficult to get the converged solutions during LFS calculation. In this study, the LFS at enginerelevant conditions are obtained from simulating propagating spherical $\mathrm{CH}_{4}$ /air flames in a closed spherical vessel. The results from different chemical mechanisms are obtained and compared. It is found that there is significant difference between the LFSs predicted from different chemical mechanisms, especially at high temperatures and pressures. It is also shown that the reduced mechanism has very good performance in terms of predicting the LFS at engine-relevant conditions. Besides, the performance of difference chemical mechanisms in terms of predicting the ignition delay time and the excitation time is assessed and significant difference is also observed.
\end{abstract}

\section{INTRODUCTION}

The combustion process in the spark ignition engines (SIEs) involves complex interactions between fluid dynamic, thermodynamic and chemical processes (Gillespie et al., 2000). In order to improve the engine design and achieve highefficiency and low-emission, it requires a fundamental understanding of combustion process in SIEs. Numerical simulations of the turbulent premixed combustion are nowadays an essential tool to investigate the combustion process in SIEs.

In certain turbulent premixed combustion modelling for SIEs, the laminar flame speed (LFS) is used as an input for the evaluation of turbulent flame speed. For example, the flamelet model assumes that the characteristic combustion time is shorter than the turbulent eddy turn-over time such that the reaction takes place in relatively thin layers and the inner flame structure is close to a laminar flame (Poinsot and
Veynante, 2005). Under this assumption, the LFS is used as an input to model the turbulent burning rate by considering the enlargement of flame surface by turbulence (Duclos et al., 1993). Therefore, the LFS is important for modelling turbulent premixed combustion in SIEs.

The LFS is a fundamental physicochemical property of a combustible mixture. It depends on the local pressure, unburned gas temperature and equivalence ratio. Usually the change of LFS with the pressure, unburned gas temperature and equivalence ratio is represented by empirical correlations obtained from fitting of experimental or numerical LFS data (Amirante et al., 2017). Such empirical correlations can be easily implemented modelling combustion in SIEs. However, the accuracy of these correlations is not warranted under engine-relevant conditions since at such conditions the experiment data reported in the literature are sparse or inconsistent (Gillespie et al., 2000). It is difficult to conduct the experiments for LFS measurement under engine-relevant conditions. For example, flame instability occurs at enginerelevant high pressures and it can significantly affect the flame propagation (Yuan et al., 2007), which prevents the accurate measurement of the LFS. Currently, the LFS at enginerelevant temperatures and pressures is usually obtained from simulations considering detailed or reduced chemical mechanisms. In the literature, there are many chemical mechanisms for the same fuel; and the LFS usually depends on the chemical kinetic model used in simulation.

Based on the above considerations, this study aims to access the performance of different chemical mechanisms on predicting LFS. Methane is considered here since it is the main component of natural gas which is widely used in SIEs, and many mechanisms have been developed for methane. The propagating spherical $\mathrm{CH}_{4} /$ air flame in a closed spherical vessel is simulated using different chemical mechanisms and the LFSs at a wide range of engine-relevant conditions are obtained compared. Besides, the performance of difference chemical mechanisms in terms of predicting the ignition delay time and the excitation time is also assessed since both times are related to knock in SIEs (Bates et al., 2017). 


\section{METHODOLOGY}

\section{Numerical Setup}

One-dimensional spherical flame propagation in a closed chamber is considered. The constant-volume propagating spherical flame method (Faghih and Chen, 2016) is used to calculate the LFS. It has the advantage in obtaining the LFS at a broad range of temperature and pressure under enginerelevant conditions (Faghih and Chen, 2016). In this method, the flame is center-ignited inside a closed spherical chamber and then the pressure rise during spherical flame propagating is recorded. From the pressure history, $P=P(t)$, the local LFS, $S_{u}$, is determined according to (Faghih and Chen, 2016):

$$
S_{\mathrm{u}}=\frac{R_{\mathrm{W}}}{3}\left(1-(1-x)\left(\frac{P_{0}}{P}\right)^{1 / \gamma_{\mathrm{u}}}\right)^{-2 / 3}\left(\frac{P_{0}}{P}\right)^{1 / \gamma_{\mathrm{u}}} \frac{\mathrm{d} x}{\mathrm{~d} t}
$$

where $R_{W}$ is the inner radius of the closed chamber, $x$ the burnt mass fraction, $P_{0}$ the initial pressure, and $\gamma_{u}$ the heat capacity ratio of unburned gas. Unlike in experiments where $P(t)$ is the only available data, in simulations, the flame front position thus $x$ and $\gamma_{u}$ can be obtained at any instant. The data postprocessing is only conducted for $P>1.5 P_{0}$ so that the ignition effect is negligible.

The in-house code A-SURF (Adaptive Simulation of Unsteady Reactive Flow) (Chen et al., 2009; Chen, 2010; Dai and Chen, 2015) is used to simulate the 1D spherical flame propagation in a close chamber. The conservation equations for compressible, multi-component, reactive flow are solved using the finite volume method. CHEMKIN packages (Kee et al., 1989) are incorporated into A-SURF to calculate the chemical reaction rates and temperature-dependent thermal and transport properties. The mixture-averaged model is used to evaluate the mass diffusivities for different species. The computational domain is initially filled with static $\mathrm{CH}_{4}$ /air mixture at the initial temperature and pressure denoted as $T_{u, 0}$ and $P_{0}$, respectively. Zero flow speed and zero gradients for temperature and mass fractions are enforced at both boundaries. The flame is centrally initiated by a hot spot. It should be noted that the residence time of unburned mixture is directly related to $R_{W}$. During the spherical flame propagation, the unburned gas is compressed and it can reach very high pressure and temperature (e.g. $90 \mathrm{~atm}$ and $1100 \mathrm{~K}$ ) when the flame front is very close to the wall. Under these conditions, the end gas auto-ignition may occur, which affects the evaluation of LFS (Faghih et al., 2019). Therefore, in all simulations a small chamber radius, $R_{W}$, in the range from 5 $\mathrm{cm}$ to $0.6 \mathrm{~cm}$ is chosen so that the end gas auto-ignition dose not occur. In order to efficiently and accurately resolve the reaction zone, adaptive mesh refinement algorithm is implemented. At a high pressure up to $90 \mathrm{~atm}$, the reaction zone is always covered by the finest meshes with the width of $0.12 \mu \mathrm{m}$. Grid convergence is ensured. A-SURF has been used to simulate ignition and flame propagation in previous studies (e.g., (Chen et al., 2011; Yu and Chen, 2015)). The details on governing equations and numerical methods can be found in (Chen et al., 2009; Chen, 2010; Dai and Chen, 2015) and thereby are not repeated here.

\section{Chemical Reaction Modelling}

In the literature there are different chemical mechanisms proposed for the oxidation of methane. The performance of the following mechanisms are assessed here:

1) Aramco 3.0 Mech. with 106 species and 800 reactions (Zhou et al., 2018);

2) DTU Mech. with 68 species and 631 reactions (Hashemi et al., 2016);

3) FFCM-1 Mech. with 38 species and 291 reactions (Smith et al., 2016);

4) GRI Mech. 3.0 with 53 species and 325 reactions (Gregory et al., 2018);

5) HP Mech. with 92 species and 615 reactions (Shen et al., 2014; Zhao et al., 2017)

6) USC Mech. II with 111 species and 784 reactions (Wang et al.);

7) UCDD Mech. with 58 species and 270 reactions (UCSD, 2016).

Besides, three GRI Mech. 3.0 based skeletal or reduced mechanisms are also considered, and there contain 19 species (Lu and Law, 2008), 27 species (Chen and Chen, 2018) and 30 species (Lu and Law, 2008), respectively.

\section{RESULTS AND DISCUSSION}

\section{Results for a $\mathrm{CH}_{4} /$ air Mixture Initially at Normal Temperature and Pressure (NTP)}

Computations of one-dimensional spherical flame propagation were firstly performed for a stoichiometric $\mathrm{CH}_{4} /$ air mixture initially at $P_{0}=1 \mathrm{~atm}$ and $T_{u, 0}=300 \mathrm{~K}$ using different chemical mechanisms. Instead of comparing the pressure history on which the ignition energy has a nonnegligible influence, we show the pressure rise rate (PRR), $\mathrm{d} P / \mathrm{d} t$, as a function of the pressure in Fig. 1. In general, HP Mech predicts the highest PRR while UCSD Mech. yields the lowest value. However, it can be seen from the inset in Fig. 1 that when $P$ is below 2 atm, GRI Mech. 3.0 gives the highest prediction of PRR while FFCM-1 Mech. has the lowest prediction.

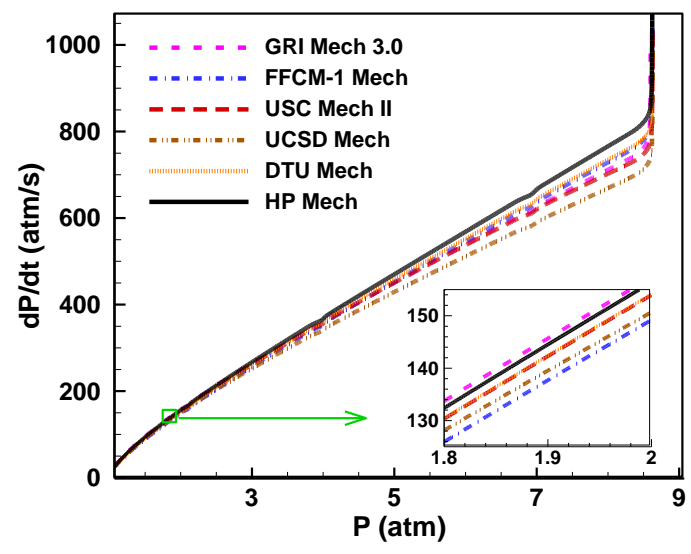

Figure 1 Pressure rise rate as a function of the instantaneous pressure for stoichiometric $\mathrm{CH}_{4}$ /air mixture initially at $P_{0}=1 \mathrm{~atm}$ and $T_{u, 0}=300 \mathrm{~K}$. 
This tendency can be observed more obviously in Fig. 2 which shows the change of $S_{u}$ with $P$. The results predicted by UCSD Mech. have a much flatter trend between $S_{u}$ and $P$ compared to those by FFCM-1 Mech. Moreover, the relative difference among $S_{u}$ predicted by different chemical mechanisms at a specified pressure is very large. At $P=1.5 \mathrm{~atm}$ and $T_{u}=336 \mathrm{~K}$, the maximum relative difference is $7.5 \%$; while it becomes $12.7 \%$ for $P=8$ atm and $T_{u}=527 \mathrm{~K}$. Therefore, even for stoichiometric $\mathrm{CH}_{4} /$ air mixture with $P_{0}=1.5 \sim 8$ atm and $T_{u}=$ $336 \sim 527 \mathrm{~K}$, the LFS strongly depends on the chemical mechanism.

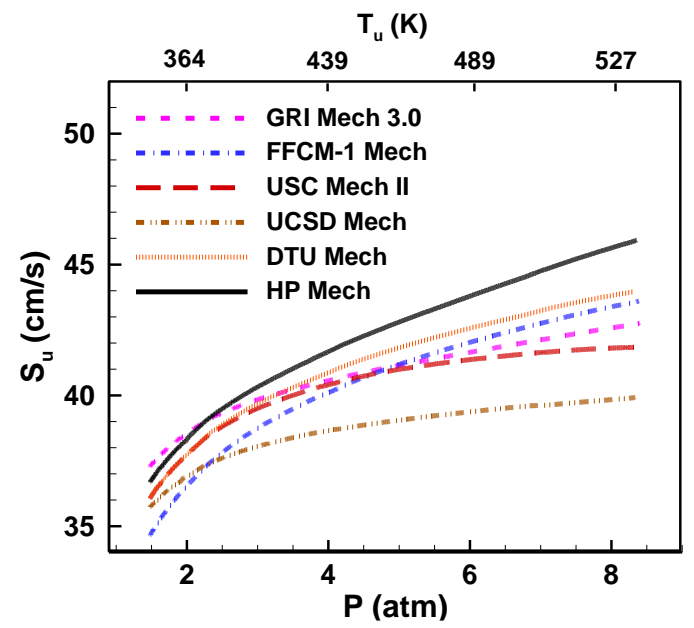

Figure 2 The LFS as a function of the instantaneous pressure and unburned gas temperature for stoichiometric $\mathrm{CH}_{4} /$ air mixture initially at $P_{0}=1 \mathrm{~atm}$ and $T_{u, 0}=300 \mathrm{~K}$.

Figure 3 compares $S_{u}$ predicted by the GRI Mech. 3.0 based skeletal or reduced mechanisms. Compared to the original GRI Mech. 3.0 with 53 species, the maximum relative difference is within $3 \%$ for the model with 27 species and it's within $1 \%$ for both models with 19 and 30 species. Therefore, $S_{u}$ can be well predicted by these three GRI Mech. 3.0 based skeletal or reduced mechanisms.

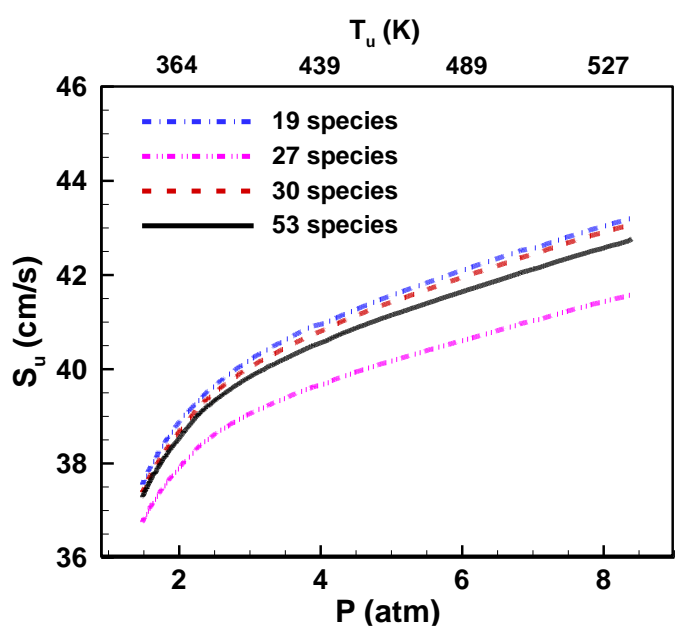

Figure 3 The LFS as a function of the instantaneous pressure and unburned gas temperature for stoichiometric $\mathrm{CH}_{4}$ /air mixture initially at $P_{0}=1$ atm and $T_{u, 0}=300 \mathrm{~K}$.

\section{Results for $\mathrm{CH}_{4} /$ air Mixtures at Engine-Relevant} Conditions

In this section, computations of 1D spherical flame propagation were performed for stoichiometric $(\phi=1)$ and fuel-lean $(\phi=0.6) \quad \mathrm{CH}_{4} /$ air mixtures at engine-relevant conditions using different chemical mechanisms.

For premixed flame propagation in SIEs, it has the pressure and unburned gas temperature in the range of 20 120 atm and 550 1050 K respectively (d'Adamo et al., 2017). To achieve such high pressure and unburned gas temperature, the unburned mixture is isentropically compressed during the spherical flame propagation.

For stoichiometric $\mathrm{CH}_{4}$ /air mixture, we performed two computations with the initial conditions at $P_{0}=10 \mathrm{~atm}$ and $T_{u, 0}=650 \mathrm{~K}$ and $P_{0}=30 \mathrm{~atm}$ and $T_{u, 0}=848 \mathrm{~K}$, respectively. These two sets of $\left(P_{0}, T_{u, 0}\right)$ are on the same isentropic-compression curve for stoichiometric $\mathrm{CH}_{4} /$ air mixture. The results are shown in Figs. 4-7.

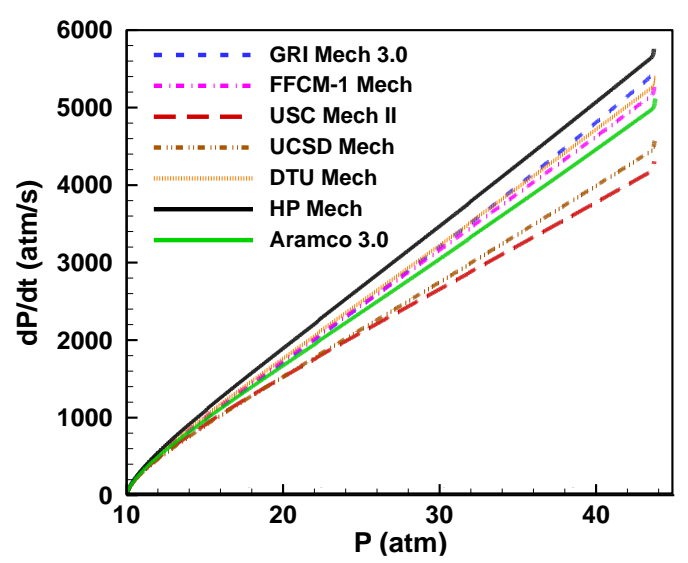

Figure 4 Pressure rise rate as a function of the instantaneous pressure for stoichiometric $\mathrm{CH}_{4}$ /air mixture initially at $P_{0}=10 \mathrm{~atm}$ and $T_{u, 0}=650 \mathrm{~K}$.

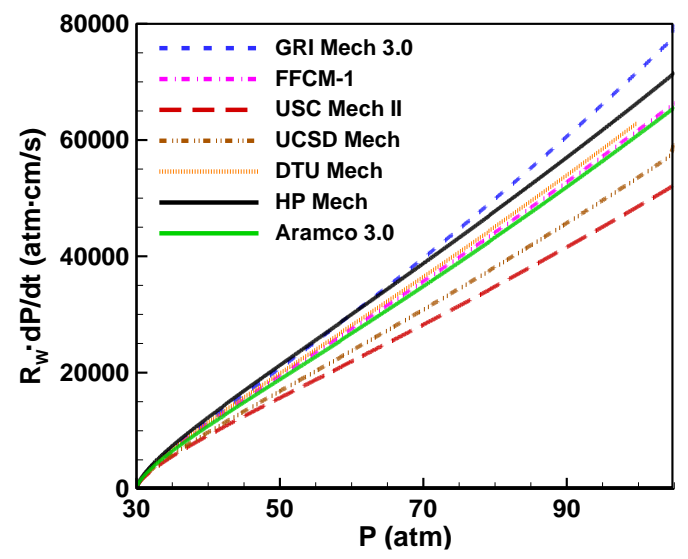

Figure 5 The product of chamber radius and pressure rise rate as a function of the instantaneous pressure for stoichiometric $\mathrm{CH}_{4} /$ air mixture initially at $P_{0}=30 \mathrm{~atm}$ and $T_{u, 0}=848 \mathrm{~K}$.

Figures 4 and 5 shows the changes of PRR with pressure. For $P_{0}=30 \mathrm{~atm}$ and $T_{u, 0}=848 \mathrm{~K}$, end gas auto-ignition occurs for large $R_{W}$ (e.g. $R_{W}=2 \mathrm{~cm}$ ). And a small value of $R_{W}$ is chosen deliberately for each mechanism to avoid end gas auto- 
ignition. The PPR is inversely proportional to the chamber radius and thereby their product, $R_{W} \cdot \mathrm{d} P / \mathrm{d} t$, is independent of $R_{W}$. Since different chamber radii were used for different mechanisms for $P_{0}=30$ atm and $T_{u, 0}=848 \mathrm{~K}$, we plot $R_{W} \cdot \mathrm{d} P / \mathrm{d} t$ instead of $\mathrm{d} P / \mathrm{d} t$ in Fig. 5. It is observed that USC Mech. II gives the lowest prediction of PRR for pressure in the range of $P=10 \sim 110 \mathrm{~atm}$. The highest prediction of PRR is given by HP Mech. and GRI Mech. 3.0 respectively for $P<60$ atm and $P>60$ atm.

Since these two initial conditions of (10 atm, $650 \mathrm{~K})$ and (30 atm, $848 \mathrm{~K}$ ) are on the same isentropic-compression curve of stoichiometric $\mathrm{CH}_{4} /$ air mixture and the unburned mixture is compressed isentropically during spherical flame propagation, the two $S_{u}-P$ curves obtained from these two computations can merge into one curve as show in Fig. 6. Similar to the trend observed in Figs. 4 and 5, the highest prediction of LFS is given by HP Mech. and GRI Mech. 3.0 respectively for $P<60$ atm and $P>60$ atm. On the other hand, USC Mech II always gives lowest LFS. The maximum relative difference among LFSs predicted by these mechanisms can reach $50 \%$. Therefore, the LFSs under engine-relevant pressures and temperatures strongly depend on the chemical mechanism using in simulation.

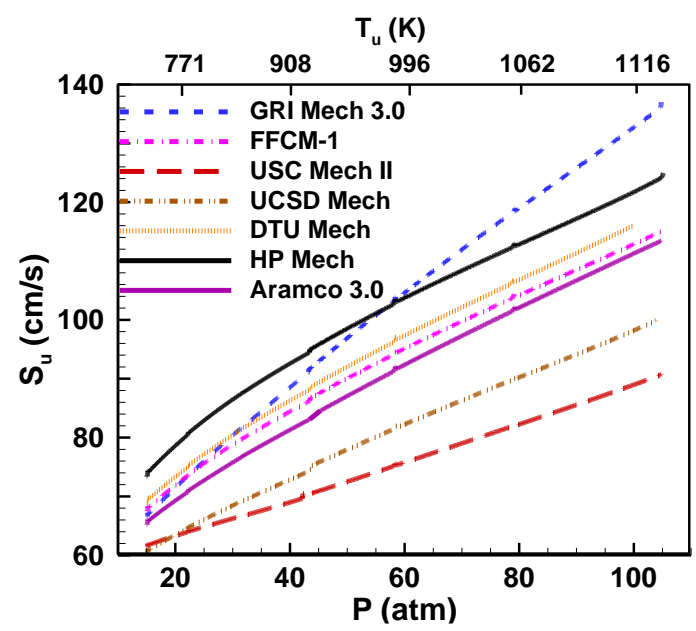

Figure 6 The LFS as a function of the instantaneous pressure and unburned gas temperature for stoichiometric $\mathrm{CH}_{4}$ /air mixture.

Besides, the performance of different GRI Mech. 3.0 based skeletal or reduced mechanisms for stoichiometric $\mathrm{CH}_{4}$ /air mixture at engine-relevant conditions was also accessed. The results were plotted in Fig. 7. The reduced model with 19 species is shown to have very good performance (within $1 \%$ ) even for $P=100 \mathrm{~atm}$ and $T_{u}=1116 \mathrm{~K}$. Compared to the original GRI Mech 3.0, the skeletal mechanism with 27 species has the under prediction within $3 \%$. Therefore, even under engine-relevant pressures and temperatures, the skeletal and reduced mechanisms have high accuracy in terms of predicting the LFS compared to the original chemical mechanism.

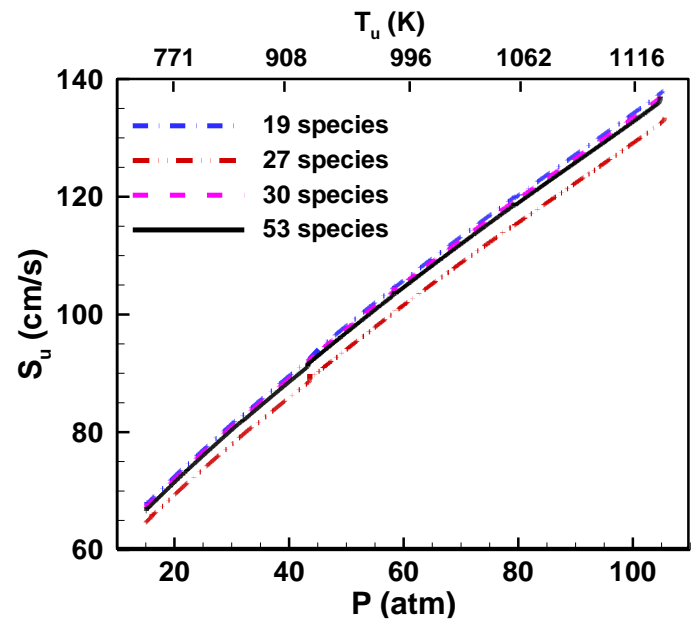

Figure 7 The LFS as a function of the instantaneous pressure and unburned gas temperature for stoichiometric $\mathrm{CH}_{4} /$ air mixture.

The results for fuel-lean $(\phi=0.6) \mathrm{CH}_{4} /$ air mixture are shown in Figs. 8 and 9. Similar trends are observed as those for the stoichiometric mixtures show in Figs. 6 and 7. At high pressure (e.g. $P>45$ atm), GRI Mech. 3.0 also gives the highest prediction of $S_{u}$. DTU Mech. and USC Mech. II gives the lowest prediction of $S_{u}$ for $P<50$ atm and $P>50$ atm respectively. The maximum relative difference among LFSs predicted by these mechanisms can reach $42 \%$. Figure 9 shows the results predicted by different GRI Mech. 3.0 based skeletal or reduced mechanisms. All the results collapse on the same curve, indicating that these three skeletal or reduced mechanisms have nearly the same prediction of LFS for fuellean $(\phi=0.6) \mathrm{CH}_{4} /$ air mixture as the original GRI Mech. 3.0.

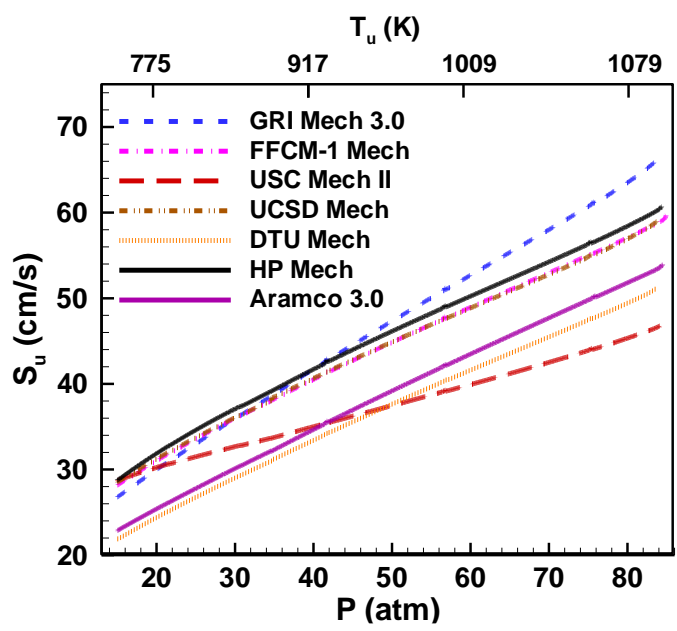

Figure 8 The LFS as a function of the instantaneous pressure and unburned gas temperature for lean $(\phi=0.6) \mathrm{CH}_{4} /$ air mixture. 


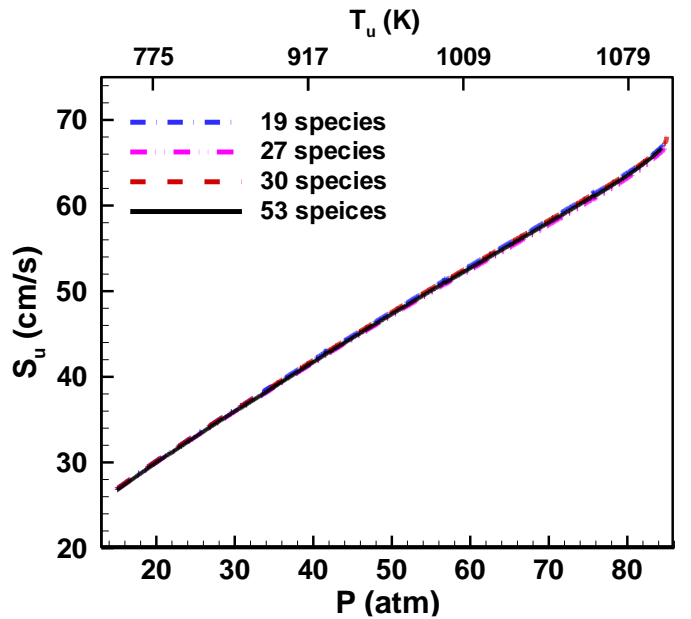

Figure 9 The LFS as a function of the instantaneous pressure and unburned gas temperature for lean $(\phi=0.6) \mathrm{CH}_{4} /$ air mixture.
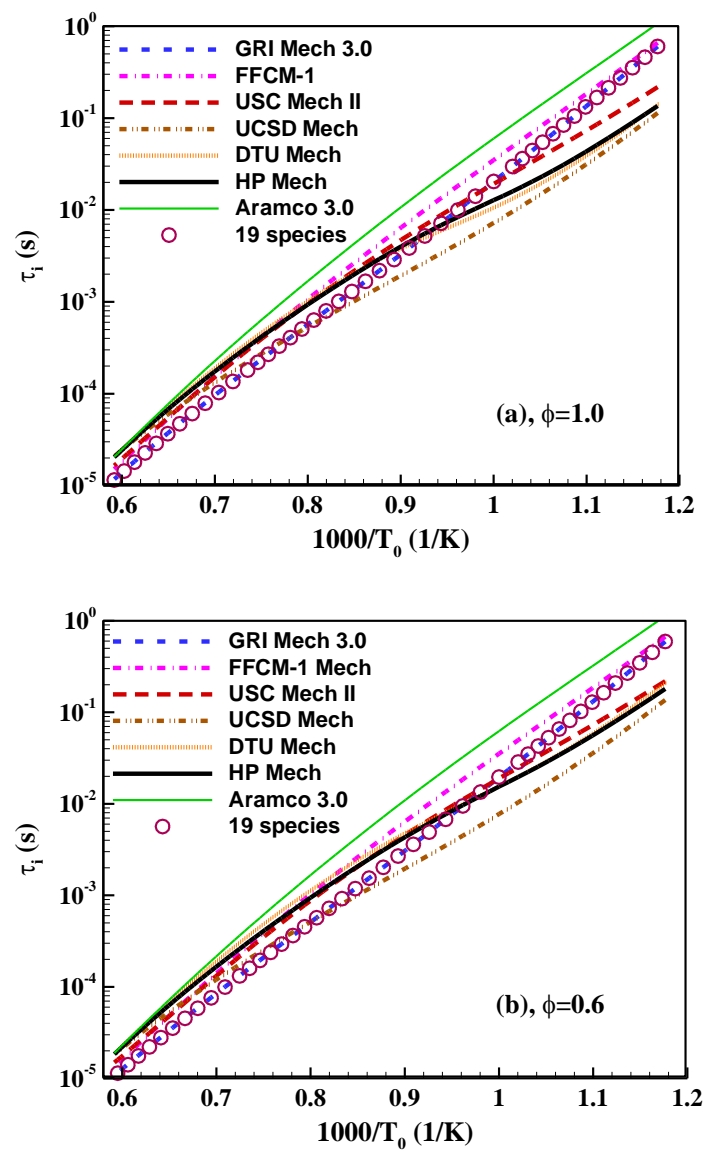

Figure 10 Change of the ignition delay time with the initial temperature for (a) stoichiometric and (b) fuel-lean $\mathrm{CH}_{4} /$ air mixtures at $\mathrm{P}=\mathbf{4 0}$ atm.

Besides the laminar flame speed, the ignition delay time, $\tau_{i}$, and the excitation time, $\tau_{e}$, were also studied since they are closely related to knocking in SIEs. The constant-pressure homogeneous ignition processes at different initial temperatures were simulated for stoichiometric $(\phi=1)$ and fuel-lean $(\phi=0.6) \quad \mathrm{CH}_{4} /$ air mixtures at $P=40$ atm using SENKIN (Lutz et al., 1988) of CHEMKIN II (Kee et al., 1989). The performance of different mechanisms were examined. The ignition delay time, $\tau_{i}$, is defined as the time to the maximum temperature rise rate; and the excitation time, $\tau_{e}$, is defined as the duration when the heat release rate is larger than $20 \%$ of the maximum heat release rate.

The results for $\tau_{i}$ and $\tau_{e}$ were plotted in Figs. 10 and 11, respectively. It is found that, Aramco 3.0 Mech. gives the highest prediction of $\tau_{i}$ while a relatively low prediction of $\tau_{e}$. HP Mech. has the lowest prediction of $\tau_{e}$. Both FFCM-1 Mech. and GRI Mech. 3.0 have relatively high prediction of $\tau_{e}$. Moreover, the relative difference among $\tau_{i}$ and $\tau_{e}$ predicted by different mechanisms are shown to increase as the initial temperature decreases. At $T_{0}=1000 \mathrm{~K}$, the ignition delay time predicted by Aramco 3.0 Mech. is around 10 times larger than that by UCSD Mech. As for the GRI Mech. 3.0 based reduced mechanism with 19 species, it has nearly the same prediction of the ignition and excitation times compared to the original GRI Mech 3.0. Therefore, the reduced mechanism can be used in modelling to greatly reduce the computational cost while achieve the same accuracy as the non-reduced mechanism.
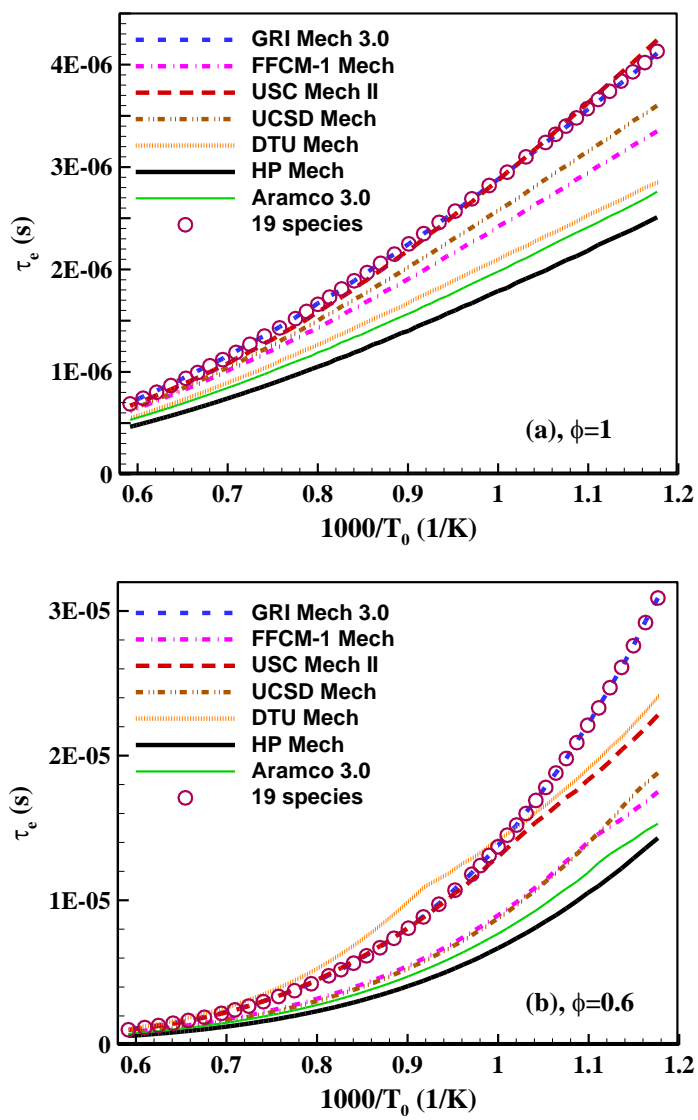

Figure 11 Change of the excitation time with the initial temperature for (a) stoichiometric and (b) fuel-lean $\mathrm{CH}_{4} /$ air mixtures at $\mathrm{P}=40$ atm.

\section{CONCLUSIONS}

In this study, the propagating spherical $\mathrm{CH}_{4} /$ air flames in a closed spherical vessel were simulated using different chemical mechanisms reported in the literature. The LFS at a wide range of pressures and temperatures within the enginerelevant conditions were obtained through the constant volume spherical flame method. The stoichiometric $(\phi=1)$ and 
lean $(\phi=0.6) \mathrm{CH}_{4}$ /air mixtures were both considered. It was found that the LFSs under engine-relevant pressures and temperatures strongly depend on the chemical mechanism using in simulation. The maximum relative difference among LFSs predicted by different mechanisms can reach $50 \%$. It was also shown that even under engine-relevant conditions, the skeletal and reduced mechanisms have high accuracy in terms of predicting the LFS compared to the original chemical mechanism.

In addition, the performance of different chemical mechanisms in terms of predicting the ignition delay time and excitation time was also accessed. Large discrepancies in the ignition delay times and excitation times were also observed. The above results indicate that even for the simplest hydrocarbon fuel, methane, great effort still needs to be devoted to developing chemical mechanisms with accurate prediction of ignition and flame propagation under enginerelevant conditions.

\section{ACKNOWLEDGMENTS}

This work was supported by National Natural Science Foundation of China (Nos. 91741126, 51861135309 and 91541204). We thank Professor Chongwen Zhou at Beihang University for providing us the Aramco 3.0 with 106 species and 800 reactions (only for high temperature chemistry).

\section{REFERENCES}

Amirante R, Distaso E, Tamburrano P, et al. (2017) Laminar flame speed correlations for methane, ethane, propane and their mixtures, and natural gas and gasoline for spark-ignition engine simulations. International Journal of Engine Research 18: 951-970. doi:10.1016/j.proci.2006.07.180

Bates L, Bradley D, Gorbatenko I, et al. (2017) Computation of methane/air ignition delay and excitation times, using comprehensive and reduced chemical mechanisms and their relevance in engine autoignition. Combustion and Flame 185: 105-116. doi:10.1016/j.combustflame.2017.07.002

Chen Y and Chen J-Y. (2018) Towards improved automatic chemical kinetic model reduction regarding ignition delays and flame speeds. Combustion and Flame 190: 293301. doi:10.1016/j.combustflame.2017.11.024

Chen Z. (2010) Effects of radiation and compression on propagating spherical flames of methane/air mixtures near the lean flammability limit. Combustion \& Flame 157: 22672276. doi:10.1016/j.combustflame.2010.07.010

Chen Z, Burke MP and Ju Y. (2009) Effects of Lewis number and ignition energy on the determination of laminar flame speed using propagating spherical flames. Proceedings of the Combustion Institute 32: 1253-1260. doi:10.1016/j.proci.2008.05.060

Chen Z, Burke MP and Ju Y. (2011) On the critical flame radius and minimum ignition energy for spherical flame initiation. Proceedings of the Combustion Institute 33: 12191226. doi:10.1016/j.proci.2010.05.005

d'Adamo A, Del Pecchia M, Breda S, et al. (2017) Chemistry-Based Laminar Flame Speed Correlations for a Wide Range of Engine Conditions for Iso-Octane, n-Heptane,
Toluene and Gasoline Surrogate Fuels. SAE Technical Paper. doi:10.4271/2017-01-2190

Dai P and Chen Z. (2015) Supersonic reaction front propagation initiated by a hot spot in n-heptane/air mixture with multistage ignition. Combustion and Flame 162: 41834193. doi:10.1016/j.combustflame.2015.08.002

Duclos J, Veynante D and Poinsot T. (1993) A comparison of flamelet models for premixed turbulent combustion. Combustion and Flame 95: 101-117. doi:10.1016/00102180(93)90055-8

Faghih M and Chen Z. (2016) The constant-volume propagating spherical flame method for laminar flame speed measurement. Science Bulletin 61: 1-15.

Faghih M, Li H, Gou X, et al. (2019) On laminar premixed flame propagating into autoigniting mixtures under enginerelevant conditions. Proceedings of the Combustion Institute 37: 4673-4680. doi:10.1016/j.proci.2018.06.058

Gillespie L, Lawes M, Sheppard C, et al. (2000) Aspects of laminar and turbulent burning velocity relevant to SI engines. Sae Transactions: 13-33.

Gregory P, Golden D, Frenklach M, et al. (2018) GRIMech 3.0 (Tech. Rep.). UC Berkeley.

Hashemi H, Christensen JM, Gersen S, et al. (2016) Highpressure oxidation of methane. Combustion and Flame 172: 349-364. doi:10.1016/j.combustflame.2016.07.016

Kee RJ, Rupley FM and Miller JA. (1989) Chemkin-II: A Fortran chemical kinetics package for the analysis of gasphase chemical kinetics. Sandia National Labs., Livermore, CA (USA).

Lu T and Law CK. (2008) A criterion based on computational singular perturbation for the identification of quasi steady state species: A reduced mechanism for methane oxidation with NO chemistry. Combustion and Flame 154: 761-774. doi:10.1016/j.combustflame.2008.04.025

Lutz AE, Kee RJ and Miller JA. (1988) SENKIN: A FORTRAN program for predicting homogeneous gas phase chemical kinetics with sensitivity analysis. Sandia National Labs., Livermore, CA (USA).

Poinsot T and Veynante D. (2005) Theoretical and numerical combustion: RT Edwards, Inc.

Shen X, Yang X, Santner JS, et al. (2014) Experimental and kinetic studies of acetylene flames at elevated pressures. 52nd Aerospace Sciences Meeting. 0129.

Smith G, Tao Y and Wang H. (2016) Foundational Fuel Chemistry Model Version 1.0 (FFCM-1). epub, accessed July 26: 2018.

UCSD. (2016) Chemical-Kinetic Mechanisms for Combustion Applications. Available at: http://web.eng.ucsd.edu/mae/groups/combustion/mechanism. html.

Wang H, You X, Joshi A, et al. High-Temperature Combustion Reaction Model of H 2/CO/C 1-C 4 Compounds, May 2007.

$\mathrm{Yu} \mathrm{H}$ and Chen Z. (2015) End-gas autoignition and detonation development in a closed chamber. Combustion and Flame 162: 4102-4111. doi:10.1016/j.combustflame.2015.08.018

Yuan J, Ju Y and Law CK. (2007) On flame-front instability at elevated pressures. Proceedings of the 
$\begin{array}{llll}\text { Combustion } \quad \text { Institute } & 31: & 1267-1274 .\end{array}$ doi:10.1016/j.proci.2006.07.180

Zhao H, Fu J, Haas FM, et al. (2017) Effect of prompt dissociation of formyl radical on 1, 3, 5-trioxane and $\mathrm{CH} 2 \mathrm{O}$ laminar flame speeds with $\mathrm{CO} 2$ dilution at elevated pressure. Combustion and Flame 183: 253-260. doi:10.1016/j.combustflame.2017.05.005

Zhou C-W, Li Y, Burke U, et al. (2018) An experimental and chemical kinetic modeling study of 1, 3-butadiene combustion: Ignition delay time and laminar flame speed measurements. Combustion and Flame 197: 423-438. doi:10.1016/j.combustflame.2018.08.006 